\title{
Physical Exertion at Work and Addictive Behaviors: Tobacco, Cannabis, Alcohol, Sugar and Fat Consumption: Longitudinal Analyses in trhe CONSTANCES Cohort
}

\section{Nadine Hamieh ( $\square$ nadine.hamieh@inserm.fr )}

INSERM, Population-based Epidemiological Cohorts Unit, UMS 011

\section{Alexis Descatha}

Academic Hospital CHU Angers

\section{Marie Zins}

INSERM, Population-based Epidemiological Cohorts Unit, UMS 011

\section{Marcel Goldberg}

INSERM, Population-based Epidemiological Cohorts Unit, UMS 011

\section{Sébastien Czernichow}

Université de Paris, AP-HP, Hôpital européen Georges-Pompidou

\section{Nicolas Hoertel}

Université de Paris, AP-HP, Hôpital Corentin-Celton, Issy-les-Moulineaux, INSERM, Institut de Psychiatrie et Neurosciences de Paris (IPNP), UMR_S1266

\section{Marie Plessz}

INRAE, Centre Maurice Halbwachs (ENS, EHESS, CNRS) UMR 8097

\section{Yves Roquelaure}

Academic Hospital CHU Angers

\section{Frédéric Limosin}

Université de Paris, AP-HP, Hôpital Corentin-Celton, Issy-les-Moulineaux, INSERM, Institut de Psychiatrie et Neurosciences de Paris (IPNP), UMR_S1266

\section{Cédric Lemogne}

Université de Paris, AP-HP, Hôpital Hôtel-Dieu, INSERM, Institut de Psychiatrie et Neurosciences de Paris (IPNP), UMR_S1266

\section{Joane Matta}

INSERM, Population-based Epidemiological Cohorts Unit, UMS 011

\section{Guillaume Airagnes}

Université de Paris, AP-HP.Centre-Université de Paris, DMU Psychiatrie et Addictologie

\section{Research Article}

Keywords: Physical exertion , work and addictive behaviors, tobacco, cannabis, alcohol, sugar and fat consumption 
Posted Date: August 3rd, 2021

DOI: https://doi.org/10.21203/rs.3.rs-757811/v1

License: (c) (i) This work is licensed under a Creative Commons Attribution 4.0 International License. Read Full License

Version of Record: A version of this preprint was published at Scientific Reports on January 13th, 2022. See the published version at https://doi.org/10.1038/s41598-021-04475-2. 


\section{Abstract}

We examined the prospective association of physical exertion at work with subsequent tobacco, cannabis, alcohol use, and sugar and fat consumption. Volunteers of the French population-based CONSTANCES cohort currently employed were included from 2012-2017 for tobacco and cannabis outcomes ( $n=100,612$ ), and from 2012-2016 for alcohol and sugar and fat outcomes $(n=75,414)$. High level of physical exertion at work was defined as a score $\geq 12$ at the Rating Perceived Exertion Borg scale. Substance use was self-reported and diet rich in sugar and fat was obtained from principal component analysis and analyzed as quartiles. Generalized linear models computed odds of substance use and sugar and fat consumption at follow-up according to baseline physical exertion at work, while adjusting for sociodemographic factors, depressive symptoms and baseline level of consumption. High physical exertion was associated with tobacco use with dose-dependent relationships. It was also associated with increased odds of cannabis use at least once per month compared to no use in the past and with increased odds of diet rich in sugar and fat. Hence, the role of physical exertion at work on tobacco and cannabis use and diet rich in sugar and fat should be tackled for information and prevention strategies.

\section{Introduction}

Substance use are the first preventable cause of premature death worldwide ${ }^{1}$. If left untreated, they could lead to somatic disorders (e.g., cancers and cardiovascular disorders) ${ }^{2,3}$, psychiatric disorders (e.g., mood disorders and suicide) ${ }^{4-7}$ and social deprivation including occupational issues (e.g., absenteeism, work accident and job loss) 8,9 . At the population level, the most concerned substances are tobacco, cannabis and alcohol use. Among former substance users, prevention of relapse is also a major issue ${ }^{10}$. Sugar and fat overconsumption are also highly prevalent in western countries and they share common vulnerability factors with substance use ${ }^{9}$.

Substance use and sugar and fat consumption could be driven by environmental factors such as occupational factors ${ }^{11}$. For instance, substance use and craving for sugar and fat increase after losing a job ${ }^{12}$ or retiring ${ }^{13}$. However, work stress may increase the likelihood of substance use and relapse in former users ${ }^{14}$. For instance, high job demand has been associated with increased risks of using substances in the workplace ${ }^{15}$. In this study, we focused on physical exertion at work and its associations with tobacco, cannabis and alcohol use and high sugar and fat consumption. Such working conditions have already been associated with detrimental physical health consequences (e.g. musculoskeletal disorders) ${ }^{16}$. However, its potential consequences on substance use and sugar and fat consumption have not been examined yet, to the best of our knowledge. Since occupational health strategies exist to deal with physical exertion at work, their benefits could be extended to decreasing the burden of such detrimental behaviors.

We took advantage of the CONSTANCES French national population-based cohort to examine prospectively the relations between physical exertion at work and tobacco, cannabis, alcohol use and sugar and fat consumption in a large sample of employees randomly recruited from various social and occupational backgrounds ${ }^{17}$. In addition, sociodemographic factors and depressive symptoms were also reported, allowing to take into account their potential confounding and/or moderating roles. We hypothesized that physical exertion at work is associated with increased substance use and sugar and fat consumption. Since gender and age are related to different patterns of substance use, sugar and fat consumption and occupational factors ${ }^{18}$, we also planned to examine changes in this association according to sex and age groups. 


\section{Methods}

\section{Study design}

The French population-based CONSTANCES cohort enrolled volunteers since 2012, aged 18-69 years at baseline, and according to a random sampling scheme stratified on age, gender, socioeconomic status and region of France ${ }^{17}$. Among the different procedures conducted with participants, they completed annual self-administered questionnaires on their lifestyle, health, social, and personal characteristics. All the procedures are detailed at www.constances.fr. CONSTANCES has obtained the authorization of the National Data Protection Authority, was approved by the Institutional Review Board of the National Institute for Medical Research (Authorization number 910486) and was performed in accordance with relevant guidelines and regulations. All participants signed an informed consent form to be included in the cohort.

\section{Study populations}

A total of 199,717 volunteers were enrolled in the CONSTANCES cohort between January 6, 2012 and January 8, 2020. Among these volunteers, those who were not employed at baseline $(n=62,581)$ were excluded by selecting those who reported to have currently a job. Since outcomes were available at different periods of follow-ups, individuals included after January 1, $2018(n=36,524)$ were excluded when studying the tobacco and cannabis outcomes, to allow for one-year of follow-up duration (since the last follow-up date of these outcomes was in 2018 at the time the present study was conducted). Regarding alcohol and sugar and fat outcomes, volunteers included after January 2017 ( $n=61,722)$ were excluded since the last available follow-up endpoint was in 2017 for these outcomes. Data on sugar and was available only at baseline and five years later in 2017. Hence, a total of 100,612 participants were included for studying tobacco and cannabis use and 75,414 for studying alcohol use and diet rich in sugar and fat (Fig. 1).

\section{Assessment of physical exertion}

Physical exertion was assessed using the Rating Perceived Exertion (RPE) Borg scale at baseline. The RPE Borg scale is a scale ranging from 6-20 with 6 'No effort at all' and 20 'Exhausting' that assesses the perceived physical exertion by asking the volunteers to rate their intensity of physical effort during a typical working day ${ }^{19}$. Volunteers with a score of $\geq 12$ were considered to be exposed to high physical exertion according to the 'French National Research and Safety Institute for the Prevention of Occupational Accidents and Disease' 20 .

\section{Assessment of tobacco use}

Smoking status (i.e., Never smokers; Former smokers; Current smokers) was self-reported, as well as daily tobacco consumption in number of cigarettes per day among current smokers. Thus, we further categorized the current smokers into three categories: Current light smokers (< 10 cigarettes/day); Current moderate smokers (1018 cigarettes/day) and Current heavy smokers (> 19 cigarettes/day) ${ }^{18}$ and computed a variable with 5 modalities as followed: Non-smokers; Ex-smokers; Current light smokers; Current moderate smokers and Current heavy smokers.

Since we also planned to examine the risk of relapse, the following variables were also computed:

- 'Relapse of tobacco use among ex-smokers at baseline' with 'No': remained non-smokers at follow-up and 'Yes': became current smokers at follow-up irrespective of the tobacco use intensity. 
- 'Changing status among current smokers at baseline' with 'Ex-smokers': stopped smoking at follow-up; 'Current light smokers': remained current light smokers at follow-up; 'Current moderate smokers': remained current moderate smokers at follow-up and 'Current heavy smokers': remained current heavy smokers at follow-up

- 'Changing status among ever smokers (i.e., former smokers and current smokers) at baseline with: 'Smokers at baseline and follow-up': remained current smokers at follow-up irrespective of the consumption; 'Smokers at baseline and stopped at follow-up': became ex-smokers at follow-up; ex-smokers at baseline and follow-up and "Ex-smokers at baseline and started smoking at follow-up'.

- Changes in number of cigarettes per day among continuous current smokers, computed as the difference between number of cigarettes per day at follow-up and at baseline.

\section{Assessment of cannabis use}

From three questions assessing the frequency of cannabis use at follow-up among no cannabis users for at least 12 months at baseline, the following categorical variable was computed: no use in the past 12 months at both assessments (reference category), cannabis use less than once a month at follow-up, cannabis use at least once per month at follow-up.

\section{Assessment of alcohol use}

Alcohol use was assessed continuously and categorically. It was assessed continuously as the difference between the weekly consumption at follow-up and at baseline. The weekly consumption was computed in drinks per week at baseline and at follow-up based on a reporting of all the alcoholic beverages consumed the previous week by multiplying the daily number of drinks per day by 7 . Then, alcohol use was assessed categorically according to the World Health Organization risk level classification (WHO, 2000): "Low risk" (1-27 drinks/week in men and 1-13 in women); "No use" and "At risk" ( $\geq 28$ drinks/week in men and $\geq 14$ in women).

\section{Assessment of diet rich in sugar and fat}

Diet rich in sugar and fat was assessed using the principal component analysis (PCA) of a 32-item qualitative food frequency questionnaire. These items represented the daily frequency of the consumed food (i.e., sugar, meat, cheese, yogurt and others) on a scale from $0-4$ with 0 being 'rarely or never' and 4 ' 5 or more'. From these items, three factors were generated: diet rich in sugar and fat, traditional diet and diet rich in low fat protein (Supplementary Table S1). Diet rich in sugar and fat which was our variable of interest, was assessed as quartiles variables using the indices obtained by the principal component analysis while considering the first quartile as the reference group.

\section{Assessment of covariables at baseline}

Sociodemographic factors included age, sex, occupational grade (low: manual and clerical; medium: technical; high: managerial positions), educational level and household income. Educational level and household income were assessed using self-reported questions on the highest obtained diploma based on the International Standard Classification of Education $2011^{21}$, and on total household net monthly income, respectively.

Depressive symptoms were assessed using the French version of the CES-D scale which has a high internal consistency ( $a=0.90$ in the CONSTANCES cohort) and volunteers with a score of $\geq 19$ were considered to be 
clinically depressed according to the validated threshold for the French version (sensitivity and specificity $>85 \%$ for the diagnosis of major depression) ${ }^{22}$.

\section{Statistical analysis}

Generalized linear regressions were computed to study the associations between physical exertion at work (exposure) and tobacco, cannabis, alcohol use and diet rich in sugar and fat (outcomes). All the analyses were carried by using unadjusted and fully-adjusted models. The fully-adjusted models were adjusted for all the covariables mentioned above in addition to the baseline level of consumption for the substance chosen as the outcome. However, regarding the changes in tobacco and cannabis use between follow-up and baseline, there was no adjustment for baseline level of consumption since the construction of the outcome variable already included this information. Cochran-Mantel-Haenszel tests were performed to search for trends between physical exertion and the outcomes.

Then, the analyses were stratified for sex and age, separately. The following age groups were considered: 18-29; 30-39; 40-49; 50-59; $\geq 60$.

Lastly, sensitivity analyses were performed for diet rich in sugar and fat since this outcome had only one available follow-up endpoint, 2017 and was assessed over 5 years. First, we additionally adjusted for duration of follow-up in the model. Duration of follow-up was assessed continuously as the difference between 2017 and at baseline. Second, we tested for statistical interaction between physical exertion at work and duration of follow-up since duration of follow-up could be an effect modifier between the association between physical exertion and diet rich in sugar and fat. If a significant interaction was found, stratified analyses were done.

Missing data were handled by multiple imputations. All $P$-values were two-sided with an a $=0.05$. All statistical analyses were undertaken using the SAS system software (version 9.4, SAS Institute, Cary, NC).

\section{Results}

The baseline characteristics of the 100,612 and 75,414 employees included between 2012-2017 (to study tobacco and cannabis use) and 2012-2016 (to study alcohol use and diet rich in sugar and fat), are respectively summarized in Table 1. The prevalence of high physical exertion at work was $33.3 \%$ among employees included between 2012-2017 $(n=33,579)$ and 32.8\% among those included between 2012-2016 $(n=24,795)$. All the covariables were associated with physical exertion at work. For instance, employees with higher physical exertion were older, more likely to be men, had a lower occupational grade, a lower education and a lower income and less likely to report depressive symptoms (all $P<0.0001$ ). 
Table 1

Baseline characteristics of 100,612 and 75,414 employees included between 2012-2017 (to study tobacco and cannabis use) and 2012-2016 (to study alcohol use and diet rich in sugar and fat), respectively by exposure to physical exertion in the CONSTANCES cohort study

\begin{tabular}{|c|c|c|c|c|c|c|c|c|}
\hline & \multicolumn{3}{|c|}{$\begin{array}{l}\text { Between 2012- } \\
2017\end{array}$} & \multirow[b]{2}{*}{$P$} & \multirow[b]{2}{*}{ Total } & \multicolumn{2}{|c|}{$\begin{array}{l}\text { Between 2012- } \\
2016\end{array}$} & \multirow[b]{2}{*}{$P$} \\
\hline & Total & $\begin{array}{l}\text { High } \\
\text { physical } \\
\text { exertion }\end{array}$ & $\begin{array}{l}\text { No high } \\
\text { physical } \\
\text { exertion }\end{array}$ & & & $\begin{array}{l}\text { High } \\
\text { physical } \\
\text { exertion }\end{array}$ & $\begin{array}{l}\text { No high } \\
\text { physical } \\
\text { exertion }\end{array}$ & \\
\hline & $\begin{array}{l}N= \\
100,612\end{array}$ & $\begin{array}{l}N= \\
33,579\end{array}$ & $\begin{array}{l}N= \\
67,033\end{array}$ & & $\begin{array}{l}N= \\
75,414\end{array}$ & $\begin{array}{l}N= \\
24,795\end{array}$ & $\begin{array}{l}N= \\
50,619\end{array}$ & \\
\hline $\begin{array}{l}\text { Mean (SD) age, } \\
\text { years }\end{array}$ & $\begin{array}{l}43.6 \\
(10.9)\end{array}$ & $\begin{array}{l}43.4 \\
(11.2)\end{array}$ & $\begin{array}{l}43.6 \\
(10.8)\end{array}$ & $\begin{array}{l}< \\
0.0001\end{array}$ & $\begin{array}{l}43.9 \\
(10.9)\end{array}$ & $\begin{array}{l}43.7 \\
(11.2)\end{array}$ & $\begin{array}{l}43.9 \\
(10.8)\end{array}$ & $\begin{array}{l}< \\
0.0001\end{array}$ \\
\hline Age, $\%$ & & & & $\begin{array}{l}< \\
0.0001\end{array}$ & & & & $\begin{array}{l}< \\
0.0001\end{array}$ \\
\hline $18-29$ & 13.0 & 38.0 & 62.0 & & 12.6 & 37.6 & 62.4 & \\
\hline $30-39$ & 24.7 & 30.6 & 69.4 & & 23.8 & 30.0 & 70.0 & \\
\hline $40-49$ & 29.9 & 33.0 & 67.0 & & 29.9 & 32.7 & 67.3 & \\
\hline $50-59$ & 26.5 & 34.9 & 65.1 & & 27.6 & 34.1 & 65.9 & \\
\hline$\geq 60$ & 5.9 & 30.0 & 70.0 & & 6.1 & 29.7 & 70.3 & \\
\hline Male sex, \% & 47.0 & 34.5 & 65.5 & $\begin{array}{l}<.0001 \\
0.00\end{array}$ & 47.3 & 33.8 & 66.2 & $\begin{array}{l}<.0001 \\
0.00\end{array}$ \\
\hline $\begin{array}{l}\text { Occupational } \\
\text { grade, \% }\end{array}$ & & & & $\begin{array}{l}< \\
0.0001\end{array}$ & & & & $\begin{array}{l}< \\
0.0001\end{array}$ \\
\hline Low & 38.1 & 50.7 & 49.3 & & 37.5 & 50.5 & 49.5 & \\
\hline Medium & 29.1 & 36.4 & 63.6 & & 29.3 & 35.6 & 64.4 & \\
\hline High & 32.8 & 10.6 & 89.4 & & 33.2 & 10.6 & 89.4 & \\
\hline $\begin{array}{l}\text { Educational } \\
\text { level, \% }\end{array}$ & & & & $\begin{array}{l}< \\
0.0001\end{array}$ & & & & 0.0001 \\
\hline$<$ Baccalaureate & 35.9 & 53.2 & 46.8 & & 36.5 & 52.0 & 48.0 & \\
\hline$\geq$ Baccalaureate & 64.1 & 22.3 & 77.7 & & 63.5 & 21.9 & 78.1 & \\
\hline $\begin{array}{l}\text { Household } \\
\text { income, \% }\end{array}$ & & & & $\begin{array}{l}< \\
0.0001\end{array}$ & & & & < 0.0001 \\
\hline$<2100 € /$ month & 19.9 & 50.8 & 49.2 & & 19.9 & 50.5 & 49.5 & \\
\hline $\begin{array}{l}\geq 2100 \\
€ / \text { month }\end{array}$ & 80.1 & 29.0 & 71.0 & & 80.1 & 28.5 & 71.5 & \\
\hline
\end{tabular}

*Depressive symptoms were defined as having a CES-D score $\geq 19$.

Independent t-tests and Chi-square tests were computed for continuous and categorical variables, respectively. 


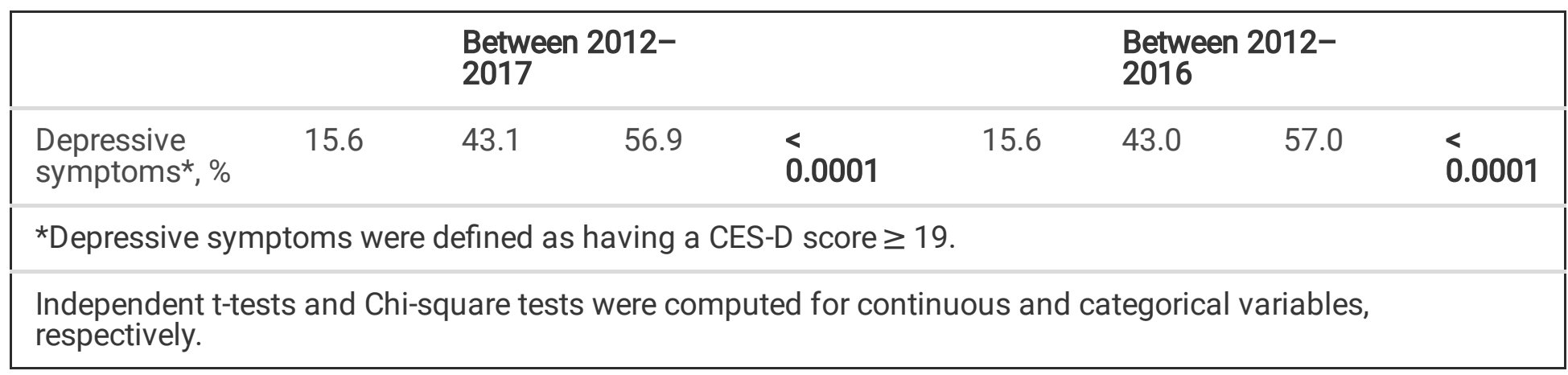

\section{Association between physical exertion at work, substance use and diet rich in sugar and fat}

\section{- Tobacco use}

In ex-smokers at baseline, high physical exertion increased the odd of relapsing (aOR: 1.13, 95\% Confidence Interval (Cl): 1.02-1.24). This association vanished in women when stratified by sex (Supplementary Table S2).

In current-smokers at baseline, high physical exertion decreased the odd of quitting (aOR: 0.78, 95\% Cl: $0.73-$ 0.84 ) (Table 2). High physical exertion increased the odd of becoming heavy smokers (aOR: 1.54, 95\%Cl: $1.33-$ 1.78). Dose-dependent relationships between physical exertion at work and tobacco use were found ( $P$-trend < $0.001)$. 
Table 2

Association between high physical exertion at work and tobacco use at one-year of follow-up among employees in the CONSTANCES cohort study, 2012-2018 (odds ratios (ORs), and 95\% confidence intervals, Cl).

\begin{tabular}{|c|c|c|c|}
\hline & & $\begin{array}{l}\text { Unadjusted } \\
\text { model }\end{array}$ & $\begin{array}{l}\text { Fully-adjusted } \\
\text { model* }\end{array}$ \\
\hline & $N(\%)$ & OR $(95 \% \mathrm{Cl})$ & OR $(95 \% \mathrm{Cl})$ \\
\hline \multicolumn{4}{|l|}{ Tobacco use } \\
\hline $\begin{array}{l}\text { Relapse of tobacco use among ex-smokers at } \\
\text { baseline }\end{array}$ & \multicolumn{2}{|l|}{30,916} & \\
\hline No & $\begin{array}{l}25,218 \\
(81.6)\end{array}$ & 1.00 & 1.00 \\
\hline Yes & $\begin{array}{l}5,698 \\
(18.4)\end{array}$ & $\begin{array}{l}1.37(1.29- \\
1.45)\end{array}$ & $1.13(1.02-1.24)$ \\
\hline Changing status among current smokers at baseline & \multicolumn{2}{|l|}{20,078} & \\
\hline Ex-smoker & $\begin{array}{l}5,787 \\
(28.8)\end{array}$ & 1.00 & 1.00 \\
\hline Current light smoker & $\begin{array}{l}8,406 \\
(41.9)\end{array}$ & $\begin{array}{l}1.54(1.43- \\
1.66)\end{array}$ & $1.21(1.12-1.31)$ \\
\hline Current moderate Smoker & $\begin{array}{l}4,751 \\
(23.7)\end{array}$ & $\begin{array}{l}2.14(1.97- \\
2.32)\end{array}$ & $1.34(1.23-1.47)$ \\
\hline Current heavy smoker & $1,134(5.6)$ & $\begin{array}{l}2.47(2.17- \\
2.81)\end{array}$ & $1.54(1.33-1.78)$ \\
\hline P-trend & \multicolumn{2}{|l|}{$<0.0001$} & \\
\hline Changing status among ever-smokers at baseline & \multicolumn{2}{|l|}{50,994} & \\
\hline $\begin{array}{l}\text { Smoker at baseline and remained smoker at follow- } \\
\text { up }\end{array}$ & $\begin{array}{l}14,291 \\
(28.0)\end{array}$ & 1.00 & 1.00 \\
\hline Smoker at baseline and stopped at follow-up & $\begin{array}{l}5,787 \\
(11.3)\end{array}$ & $\begin{array}{l}0.56(0.52- \\
0.60)\end{array}$ & $0.78(0.73-0.84)$ \\
\hline Ex-smoker at baseline and stopped at follow-up & $\begin{array}{l}25,218 \\
(49.5)\end{array}$ & $\begin{array}{l}0.60(0.58- \\
0.63)\end{array}$ & $0.85(0.81-0.90)$ \\
\hline $\begin{array}{l}\text { Ex-smoker at baseline and started smoking at follow- } \\
\text { up }\end{array}$ & $\begin{array}{l}5,698 \\
(11.2)\end{array}$ & $\begin{array}{l}0.83(0.78- \\
0.88)\end{array}$ & $0.94(0.87-1.01)$ \\
\hline \multirow[t]{2}{*}{ P-trend } & \multicolumn{2}{|l|}{$<0.0001$} & \\
\hline & & $B(95 \% \mathrm{Cl})$ & $B(95 \% \mathrm{Cl})$ \\
\hline $\begin{array}{l}\text { Number of cigarettes/day among current smokers at } \\
\text { baseline }\end{array}$ & 20,078 & $\begin{array}{l}-0.02 \\
(-0.18 ; 0.14)\end{array}$ & $0.35(0.20 ; 0.51)$ \\
\hline \multicolumn{4}{|c|}{$\begin{array}{l}\text { *Adjusted for age (years, continuous), sex, occupational grade (low; medium; high), depressive symptoms at } \\
\text { baseline (no; yes), educational level (levels, continuous) and household income (€/month, continuous). }\end{array}$} \\
\hline
\end{tabular}




\section{Unadjusted \\ model}

Fully-adjusted model*

Relapse was defined as: no (remained non-smokers at follow-up) and yes (became current smokers at followup).

Changing status among current smokers was defined as ex-smokers (stopped smoking at follow-up), current light smokers (remained current light smokers at follow-up), current moderate smokers (remained current moderate smokers at follow-up) and current heavy smokers (remained current heavy smokers at follow-up).

\section{- Cannabis use}

High physical exertion at work was associated with an increased odd of using cannabis at least once per month at follow-up in participants who were not users for the last 12 months or more (aOR: 1.31, 95\% Cl: 1.03-1.66) (Table 3). When stratified by sex, this association was lost in men (Supplementary Table S2).

Table 3

Association between high physical exertion at work and cannabis use at one-year of follow-up among employees in the CONSTANCES cohort study, 2012-2018 (odds ratios (ORs), and 95\% confidence intervals, Cl).

\begin{tabular}{|c|c|c|c|}
\hline & & $\begin{array}{l}\text { Unadjusted } \\
\text { model }\end{array}$ & $\begin{array}{l}\text { Fully-adjusted } \\
\text { model* }\end{array}$ \\
\hline & $\mathbf{N}(\%)$ & OR $(95 \% \mathrm{Cl})$ & OR $(95 \% \mathrm{Cl})$ \\
\hline \multicolumn{4}{|l|}{ Cannabis use } \\
\hline Relapse among ever-users at baseline & 34,228 & & \\
\hline $\begin{array}{l}\text { No consumption in the past } 12 \text { months at follow- } \\
\text { up }\end{array}$ & $\begin{array}{l}32,331 \\
(94.5)\end{array}$ & 1.00 & 1.00 \\
\hline In the past 12 months, $<1 /$ month & $1,558(4.5)$ & $0.90(0.80-1.00)$ & $0.93(0.82-1.06)$ \\
\hline In the past 12 months, $\geq 1 /$ month & $339(1.0)$ & $1.67(1.35-2.06)$ & $1.31(1.03-1.66)$ \\
\hline
\end{tabular}

\section{- Alcohol use}

High physical exertion was not associated with alcohol use (Table 4). 
Table 4

Association between high physical exertion at work and alcohol use at one-year of follow-up among employees in the CONSTANCES cohort study, 2012-2018 (odds ratios (ORs), and 95\% confidence intervals, Cl).

\begin{tabular}{|llll}
\hline & & Unadjusted model & Fully-adjusted model* \\
\hline & $\mathbf{N}(\%)$ & OR $(95 \% \mathrm{Cl})$ & OR $(95 \% \mathrm{Cl})$ \\
\hline Alcohol use & & & \\
\hline Low risk & $49,800(66.0)$ & 1.00 & 1.00 \\
\hline No use & $15,762(20.9)$ & $\mathbf{1 . 1 3 ( 1 . 0 9 - 1 . 1 7 )}$ & $1.01(0.97-1.06)$ \\
\hline At risk & $9,852(13.1)$ & $\mathbf{1 . 0 9 ( 1 . 0 4 - 1 . 1 4 )}$ & $1.04(0.98-1.10)$ \\
\hline & & $B(95 \% \mathrm{Cl})$ & $B(95 \% \mathrm{Cl})$ \\
\hline Number of glasses/week & 75,414 & $\mathbf{0 . 0 2 ( 0 . 1 3 ; 0 . 1 6 )}$ & $0.11(-0.03 ; 0.26)$ \\
\hline
\end{tabular}

*Adjusted for age (years, continuous), sex, occupational grade (low; medium; high), depressive symptoms at baseline (no; yes), educational level (levels, continuous), household income (€/month, continuous) and baseline level of consumption.

Alcohol use was defined as: low risk (1-27 drinks/week in men and 1-13 in women); no use and at risk ( $\geq 28$ drinks/week in men and $\geq 14$ in women).

\section{- Diet rich in sugar and fat}

High perceived physical exertion was associated with an increased odd of consuming a diet rich in sugar and fat (aOR:1.06, 95\% Cl: 1.01-1.11 and aOR: 1.13, 95\% Cl: 1.07-1.18, for the third and fourth quartiles compared to the first, respectively) (Table 5). Similar results while introducing the duration of follow-up as an additional covariable in the models, with no interaction between physical exertion and duration of follow-up.

Table 5

Association between high physical exertion at work and diet rich in sugar and fat at five years of follow-up among employees in the CONSTANCES cohort study, 2012-2018 (odds ratios (ORs), and 95\% confidence intervals, Cl).

\begin{tabular}{|c|c|c|c|}
\hline & & Unadjusted model & Fully-adjusted model* \\
\hline & $\mathbf{N}(\%)$ & OR $(95 \% \mathrm{Cl})$ & OR $(95 \% \mathrm{Cl})$ \\
\hline Diet rich in sugar and fat & & OR $(95 \% \mathrm{Cl})$ & OR $(95 \% \mathrm{Cl})$ \\
\hline First quartile & $18,704(24.8)$ & 1.00 & 1.00 \\
\hline Second quartile & $19,003(25.2)$ & $1.03(0.98-1.07)$ & $1.04(0.99-1.09)$ \\
\hline Third quartile & $18,854(25.0)$ & $1.07(1.03-1.12)$ & $1.06(1.01-1.11)$ \\
\hline Fourth quartile & $18,853(25.0)$ & $1.12(1.08-1.17)$ & $1.13(1.07-1.18)$ \\
\hline P-trend & $<0.0001$ & & \\
\hline
\end{tabular}


When stratifying by age, no differences across age categories were found regarding the significant associations between, physical exertion at work and tobacco, cannabis and alcohol use and a diet rich in sugar and fat (Supplementary Table S3).

Finally, similar findings were obtained in the non-imputed data (Supplementary Table S4).

\section{Discussion}

This study examined the prospective association between physical exertion at work and tobacco, cannabis, alcohol use, and a diet rich in sugar and fat among employees from a large population-based cohort while taking into account sociodemographic factors and depressive symptoms. Overall, high physical exertion at work was positively associated with tobacco and cannabis use, as well as with the consumptions of a diet that is rich in sugar and fat. Regarding tobacco use, when exposed to high physical exertion at work, former smokers were more likely to relapse and current smokers were more likely to increase their consumption with dose-dependent relationships.

This study has some strengths. First, we used the RPE Borg scale which is a well-validated standardized tool to assess physical exertion at work ${ }^{20}$. Second, we had the necessary data to adjust the analyses for potential confounders, and sufficient power to run stratified analyses. This study has also some limitations. First, although such a large sample of population-based workers studies a large heterogeneity of different work settings, participants in are not representative of the general population, even when randomly recruited. Thus, our results should be extrapolated with caution to other settings. Second, we cannot exclude the possibility of residual confounding since some contributing factors such as personality traits or other work factors like long-working hours, have not been measured. Third, even if we performed stratified analyses according to sociodemographic factors, we could not rule out that our associations concern only certain occupations. Fourth, the absence of quantities of fat and sugar intakes limited our ability to calculate energy intakes from these macronutrients and quantify their association with physical exertion at work. However, we do not believe this information may bias our finding as the frequency of consumption provides good information on nutrient intakes.

High physical exertion was associated with tobacco, cannabis use and diet rich in sugar and fat but not with alcohol use. Prior studies were mainly cross-sectional and none of them focused on physical exertion at work ${ }^{23-}$ 27. However, our findings are overall in accordance with prior findings showing increased risks of substance use in employees experiencing difficult working conditions (e.g., high job demand, work stress and work-related musculoskeletal disorders) ${ }^{28-30}$. We may hypothesize that work-related physical exertion could increase the need of more frequent breaks as well as close ties with peers to better cope with the intensity of work ${ }^{31}$. However, these situations may increase the likelihood of smoking ${ }^{32}$. Furthermore, physical exertion may promote sleep disorders ${ }^{33}$, and/or physical pain ${ }^{34}$, that one can try to alleviate by using cannabis. No associations were found with alcohol use. Even if this result has to be confirmed in future studies with longer duration of follow-up and measurement of alcohol use disorder, patterns of alcohol use mainly rely on other occupational factors (e.g., business lunches and machine driving) and may not be considered by employees as a good way to deal with physical exertion by seeking relaxation and rest rather than festive situations and disinhibition.

Two cross-sectional studies found that a detrimental work environment (measured by physical and biomechanical factors) was associated with obesity $\left(\mathrm{BMI} \geq 30 \mathrm{~kg} / \mathrm{m}^{2}\right)^{28,29}$. Employees who are experiencing a 
tiring job could tend to cope with this situation by increasing or modifying their eating habits into comfortable foods (with a high fat and sugar content). Moreover, access to healthy foods may not always be available or easy at work, especially when exposed to difficult working conditions, making it thus harder to optimize food intake. Finally, our findings are overall in accordance with the propensity to use substance and to overconsume sugar and fat to cope with stressful life events ${ }^{28,29,35-38}$.

When stratified by age and sex, we did not find any differences across age categories. Hence, we believe that the probability of having less healthy individuals more likely to be unemployed than are healthy individuals to be low. Hence, the associations are unlikely to be explained by a "healthy worker effect" where older employees have better resilience regarding their work exposures ${ }^{39}$.

However, we found gender differences regarding tobacco and cannabis use. The lack of association in women while studying tobacco relapse is most likely the result of a reduction in statistical power. The lack of association in men while studying cannabis use needs further studies, especially by considering other potential confounders.

In conclusion, high perceived physical exertion at work was positively associated with tobacco and cannabis use and diet rich in sugar and fat. Regarding tobacco use, dose-dependent relationships were found, and when exposed to high physical exertion at work, former smokers were more likely to relapse whereas current smokers were less likely to quit. These associations should be considered when designing preventive strategies regarding poor health outcomes associated with physical exertion at work. For example, promoting other options to have a break than smoking, informing on healthy strategies to manage sleep disorders in the workplace. Furthermore, physical exertion, which can be shortly and easily assessed in occupational health, may be an indicator of the risk of unhealthy behaviors. Thus, employees exposed to such difficult working conditions should benefit from a standardized screening for substance use and deleterious eating behaviors, and to be referred to specialized care if needed. Since the present study did not find any substantial moderating effect of sociodemographic factors and depressive symptoms, these information and prevention strategies should be spread in all the exposed employees. Future studies should examine the benefits of such interventions on reducing the likelihood of unhealthy behaviors in employees.

\section{Declarations}

\section{ACKNOWLEDGMENTS}

The authors thank the team of the "Population-based Epidemiologic Cohorts Unit" (Cohortes en population) that designed and manages the Constances Cohort Study. They also thank the National Health Insurance Fund ("Caisse nationale d'assurance maladie", CNAM) and its Health Screening Centres ("Centres d'examens de santé"), which are collecting a large part of the data, as well as the National Old-Age Insurance Fund ("Caisse nationale d'assurance vieillesse", Cnav) for its contribution to the constitution of the cohort, ClinSearch, Asqualab and Eurocell, which are conducting the data quality control.

\section{AUTHOR CONTRIBUTIONS}

All authors substantially contributed to the writing of the article and have approved the manuscript and submission to Scientific Reports. 


\section{FUNDING SOURCES}

Nadine Hamieh was supported by a grant from "Direction de la recherche, des études, de l'évaluation et des statistiques", DREES, Ministry of Labour, France. The CONSTANCES Cohort Study was supported and funded by the French National Health Insurance Fund ("Caisse nationale d'assurance maladie", CNAM). The CONSTANCES Cohort Study is an "Infrastructure nationale en Biologie et Santé" and benefits from a grant from the French National Agency for Research (ANR-11-INBS-0002). CONSTANCES is also partly funded by Merck Sharp \& Dohme (MSD), AstraZeneca, Lundbeck and L'Oréal. None of these funding sources had any role in the design of the study, collection and analysis of data or decision to publish.

\section{COMPETING INTERESTS}

Guillaume Airagnes declares personal fees from Pierre Fabre, Lundbeck and Pfizer, outside the submitted work. Cédric Lemogne declares personal fees from Boehringer Ingelheim, Janssen-Cilag, Lundbeck and Otsuka Pharmaceutical, outside the submitted work. Alexis Descatha declares personal fees from his mentioned affiliations, Elsevier Masson, outside the submitted work. Sebastien Czernichow declares honoraria from Novo Nordisk, Lilly France, Janssen-Cilag, Fresenius Kabi in the last 36 months and he owns share of the company MyGoodLife, outside the submitted work. The other authors declare no conflicts of interest.

\section{DATA AVAILABILITY STATEMENT}

Personal health data underlying the findings of our study are not publicly available due to legal reasons related to data privacy protection. However, the data are available upon request to all interested researchers after authorization of the French "Commission nationale de l'informatique et des libertés". The CONSTANCES email address is contact@constances.fr.

\section{References}

1. WHO. Global Health Risks: Mortality and Burden of Disease Attributable to Selected Major Risks (World Health Organization, 2009).

2. Hall, W. \& Degenhardt, L. Adverse health effects of non-medical cannabis use. The Lancet, 374, 1383-1391 (2009).

3. Corrao, G., Bagnardi, V. \& Zambon, A. \& La Vecchia, C. A meta-analysis of alcohol consumption and the risk of 15 diseases. Prev Med, 38, 613-619 https://doi.org/10.1016/j.ypmed.2003.11.027 (2004).

4. Sullivan, L. E., Fiellin, D. A. \& O'Connor, P. G. The prevalence and impact of alcohol problems in major depression: A systematic review. The American Journal of Medicine, 118, 330-341 https://doi.org/10.1016/j.amjmed.2005.01.007 (2005).

5. Wiesbeck, G. A., Kuhl, H. C., Yaldizli, Ã. \& Wurst, F. M. Tobacco Smoking and Depression - Results from the WHO/ISBRA Study., 57, 26-31 (2008).

6. Chen, V. C. H. et al. Suicide and other-cause mortality after early exposure to smoking and second hand smoking: a 12-year population-based follow-up study. PloS one, 10, e0130044 (2015). 
7. Blanco, C. et al. Cannabis Use and Risk of Psychiatric Disorders: Prospective Evidence From a US National Longitudinal Study. JAMA psychiatry, 73, 388-395 https://doi.org/10.1001/jamapsychiatry.2015.3229 (2016).

8. Shield, K. D., Rehm, M. X. \& Rehm, J. Social costs of addiction in Europe. Impact of Addictive Substances and Behaviours on Individual and Societal Well-being, 181-188(2015).

9. Morois, S. et al. Daily alcohol consumption and sickness absence in the GAZEL cohort. Eur J Public Health, 27, 482-488 https://doi.org/10.1093/eurpub/ckx012 (2017).

10. Bart, G. Maintenance medication for opiate addiction: the foundation of recovery. J Addict Dis, 31, 207-225 https://doi.org/10.1080/10550887.2012.694598 (2012).

11. Kiepek, N. \& Magalhães, L. Addictions and Impulse-Control Disorders as Occupation: A Selected Literature Review and Synthesis. Journal of Occupational Science, 18, 254-276 https://doi.org/10.1080/14427591.2011.581628 (2011).

12. Boden, J. M., Lee, J. O., Horwood, L. J., Grest, C. V. \& McLeod, G. F. Modelling possible causality in the associations between unemployment, cannabis use, and alcohol misuse. Social science \& medicine, 175, 127-134 (2017).

13. Bacharach, S., Bamberger, P. A., Sonnenstuhl, W. J. \& Vashdi, D. R. Retirement and drug abuse: The conditioning role of age and retirement trajectory. Addictive behaviors, 33, 1610-1614 (2008).

14. Sinha, R. How does stress increase risk of drug abuse and relapse?, 158, 343-359 https://doi.org/10.1007/s002130100917 (2001).

15. Frone, M. R. Prevalence and distribution of illicit drug use in the workforce and in the workplace: findings and implications from a U.S. national survey. J Appl Psychol, 91, 856-869 https://doi.org/10.1037/00219010.91.4.856 (2006).

16. Nguyen, T. H. Y. et al. Multiple Exposures and Coexposures to Occupational Hazards Among Agricultural Workers: A Systematic Review of Observational Studies. Safety and Health at Work, 9, 239-248 https://doi.org/10.1016/j.shaw.2018.04.002 (2018).

17. Zins, M., Goldberg, M. \& team, C. The French CONSTANCES population-based cohort: design, inclusion and follow-up. Eur J Epidemiol, 30, 1317-1328 https://doi.org/10.1007/s10654-015-0096-4 (2015).

18. Lohse, T., Rohrmann, S., Bopp, M. \& Faeh, D. Heavy Smoking Is More Strongly Associated with General Unhealthy Lifestyle than Obesity and Underweight. PLoS One, 11, e0148563 https://doi.org/10.1371/journal.pone.0148563 (2016).

19. Borg, G. A. Psychophysical bases of perceived exertion. Med Sci Sports Exerc, 14, 377-381 (1982).

20. INRS. (ed ED6161) (2019).

21. Schneider, S. \& The International Standard Classification of Education. 2011. Comparative Social Research 30, 365-379, doi:10.1108/S0195-6310(2013)0000030017 (2013).

22. Morin, A. J. et al. Psychometric properties of the Center for Epidemiologic Studies Depression Scale (CES-D) in French clinical and nonclinical adults. Rev Epidemiol Sante Publique, 59, 327-340 https://doi.org/10.1016/j.respe.2011.03.061 (2011).

23. Barnes, A. J. \& Zimmerman, F. J. Associations of occupational attributes and excessive drinking. Soc Sci Med, 92, 35-42 https://doi.org/10.1016/j.socscimed.2013.05.023 (2013). 
24. Zins, M. et al. Predictors of change in alcohol consumption among Frenchmen of the GAZEL study cohort., 94, 385-395 https://doi.org/10.1046/j.1360-0443.1999.9433858.x (1999).

25. Alterman, T. et al. Examining associations between job characteristics and health: linking data from the Occupational Information Network (O*NET) to two U.S. national health surveys. J Occup Environ Med, 50, 1401-1413 https://doi.org/10.1097/JOM.0b013e318188e882 (2008).

26. Marchand, A., Parent-Lamarche, A. \& Blanc, M. E. Work and high-risk alcohol consumption in the Canadian workforce. Int J Environ Res Public Health, 8, 2692-2705 https://doi.org/10.3390/ijerph8072692 (2011).

27. Marchand, A. Alcohol use and misuse: what are the contributions of occupation and work organization conditions? BMC Public Health, 8, 333 https://doi.org/10.1186/1471-2458-8-333 (2008).

28. Meneton, P. et al. Work environment mediates a large part of social inequalities in the incidence of several common cardiovascular risk factors: Findings from the Gazel cohort. Soc Sci Med, 216, 59-66 https://doi.org/10.1016/j.socscimed.2018.09.042 (2018).

29. Choi, B. et al. Sedentary work, low physical job demand, and obesity in US workers. American journal of industrial medicine, 53, 1088-1101 https://doi.org/10.1002/ajim.20886 (2010).

30. Frone, M. R. Work stress and alcohol use. Alcohol Res Health, 23, 284-291 (1999).

31. Maslach, C. \& Goldberg, J. Prevention of burnout: New perspectives. Applied and Preventive Psychology, 7, 63-74 https://doi.org/10.1016/S0962-1849(98)80022-X (1998).

32. Delaney, H., MacGregor, A. \& Amos, A. Tell them you smoke, you'll get more breaks": a qualitative study of occupational and social contexts of young adult smoking in Scotland. BMJ Open, (2018). 10.1136/ bmjopen-2018-023951

33. Ota, A. et al. Psychosocial job characteristics and insomnia: a prospective cohort study using the DemandControl-Support (DCS) and Effort-Reward Imbalance (ERI) job stress models. Sleep Med, 10, 1112-1117 https://doi.org/10.1016/j.sleep.2009.03.005 (2009).

34. Heneweer, H., Vanhees, L. \& Picavet, H. S. Physical activity and low back pain: a U-shaped relation?, 143, 2125 https://doi.org/10.1016/j.pain.2008.12.033 (2009).

35. Jarvela-Reijonen, E. et al. High perceived stress is associated with unfavorable eating behavior in overweight and obese Finns of working age. Appetite, 103, 249-258 https://doi.org/10.1016/j.appet.2016.04.023 (2016).

36. Torres, S. J. \& Nowson, C. A. Relationship between stress, eating behavior, and obesity. Nutrition, 23, 887-894 https://doi.org/10.1016/j.nut.2007.08.008 (2007).

37. Block, J. P., He, Y., Zaslavsky, A. M., Ding, L. \& Ayanian, J. Z. Psychosocial stress and change in weight among US adults. Am J Epidemiol, 170, 181-192 https://doi.org/10.1093/aje/kwp104 (2009).

38. Sinha, R. The role of stress in addiction relapse. Curr Psychiatry Rep, 9, 388-395 https://doi.org/10.1007/s11920-007-0050-6 (2007).

39. Shrank, W. H., Patrick, A. R. \& Brookhart, M. A. Healthy user and related biases in observational studies of preventive interventions: a primer for physicians. J Gen Intern Med, 26, 546-550 https://doi.org/10.1007/s11606-010-1609-1 (2011).

\section{Figures}




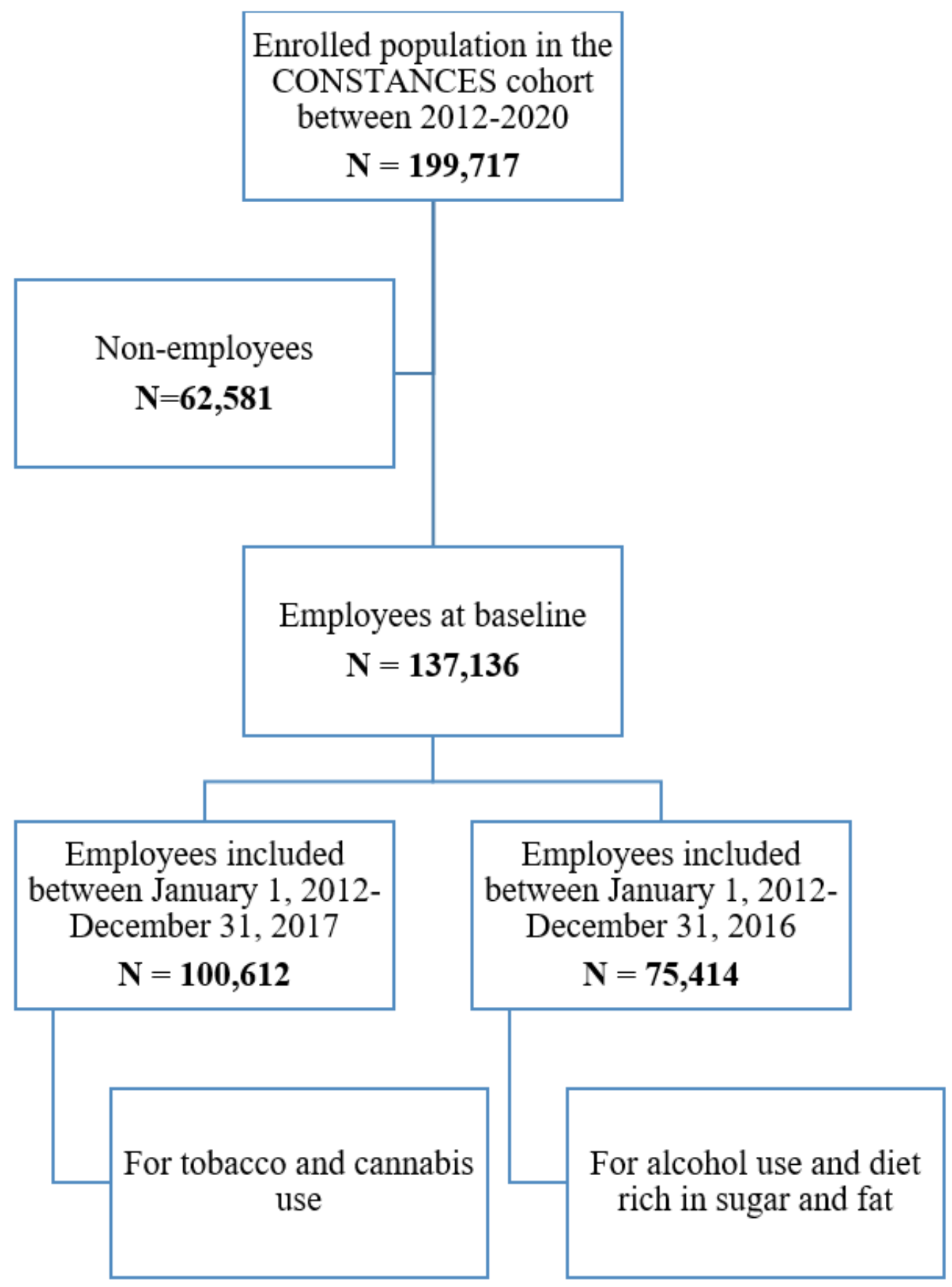

Figure 1

Cohort flow chart in the CONSTANCES cohort

\section{Supplementary Files}

This is a list of supplementary files associated with this preprint. Click to download.

- SupplementaryTableS1.docx

- SupplementaryTableS2.docx 
- SupplementaryTableS3.docx

- SupplementaryTableS4.docx 